\title{
Time out! Rethinking surgical safety: more than just a checklist
}

\author{
Matthew B Weinger (B)
}

Center for Research and Innovation in Systems Safety (CRISS) and the Department of Anesthesiology, Vanderbilt University Medical Center, Nashville, Tennessee, USA

\section{Correspondence to} Dr Matthew B Weinger, Anesthesiology/CRISS, Vanderbilt University Medical Center, Nashville, TN 37212, USA; matt.weinger@vumc.org

Accepted 16 March 2021 Published Online First 23 March 2021

\section{Sinked}

- http://dx.doi.org/10.1136/ bmjqs-2020-012001

Check for updates

(c) Author(s) (or their employer(s)) 2021. No commercial re-use. See rights and permissions. Published by BMJ.

To cite: Weinger MB. BMJ Qual Saf

2021;30:613-617.
Patients are more likely to experience preventable harm during perioperative care than in any other type of healthcare encounter. ${ }^{12}$ For several decades, a hallmark of surgical quality and safety has been the use of checklists to prevent errors (eg, wrong site surgery) and assure that key tasks have been or will be performed. The most widely used approach globally is the Surgical Safety Checklist (SSC) recommended by the WHO. ${ }^{3}$ It is divided into preinduction (or sign in, consisting of seven items performed by anaesthesia and nursing), preincision (timeout, 10 items performed by the entire team) and postsurgery (sign out, five items by the entire team). ${ }^{4}$ Most hospitals in the developed world perform the SSC or an equivalent timeout prior to surgical incision. However, preinduction briefings, and postcase debriefings in particular, are much less commonly performed. ${ }^{67}$

There are widely disseminated arguments recommending the use of checklists in healthcare ${ }^{8}$ but also recognised limitations. ${ }^{9}$ Checklist-based preincision timeouts appear to improve surgical outcomes in many settings, ${ }^{45}$ yet, in other hospitals, the introduction of the SSC failed to improve outcomes. ${ }^{10}$ Like all tools or processes intended to improve safety, ineffective implementation will reduce the desired benefits. For example, there is appreciable evidence showing that surgical teams skip or do not meaningfully respond to timeout checklist items. ${ }^{11} 12$ Even with a robust implementation, effectiveness can be weakened by contextual factors, failure of leadership or deficient safety culture.

Despite numerous studies, gaps in the evidence to guide optimal checklist use persist. For example, we do not know whether checklist-based timeouts only decrease the occurrence of the undesirable events targeted by the checklist or, as many hypothesise, whether their use also facilitates teamwork and interprofessional communication. Although there is increasing guidance on how to optimally implement checklists at the local level, many questions remain. ${ }^{13}$ Moreover, we still do not understand the circumstances in which checklist use facilitates the detection, reporting and correction of errors.

In this issue of the journal, Muensterer and colleagues ${ }^{14}$ describe a clever study in which the attending surgeon intentionally introduced errors during the preincision timeout while a medical student in the operating theatre surreptitiously noted whether the error was detected and reported by one or more members of the surgical team. If the error was not verbalised, the attending surgeon corrected the error before the timeout was complete. The single error embedded in each of 120 of 1800 paediatric operations was randomly chosen from among wrong patient name, age, gender, allergy or surgical procedure, side or site. Overall, only about half $(65 ; 54 \%)$ of all errors were detected and reported by a team member prior to surgeon correction. Of these, errors were most commonly reported by the anaesthesiologist (64\%) and almost never by residents in training $(6 \%)$ or medical students $(1 \%)$.

This study also has important limitations. Because the investigators were leading the timeouts as part of a research study, adherence to all of the checklist items was reportedly $100 \%$. Yet, few organisations consistently attain timeout adherence above 90\%. ${ }^{11}$ Since you are less likely to catch an error if you do not address that item during the timeout, in institutions with lower adherence, the proportion of missed errors may be even higher.

The authors, with input from their institutional review board, designed the study to be feasible and compliant with established human subjects protection 
principles. As such, the attending surgeon always corrected the error after the anaesthesiologist's component of the timeout but before the nurses' component. By excluding the part of the timeout when the nurses address their checklist items (eg, instruments are sterile,) followed by a final opportunity as the timeout ends to note any errors or concerns, the study may have underestimated the rate of error reporting.

Because the study did not query team members individually after the timeout, we also do not know how many errors were detected but not annunciated. For example, recognised errors that were attributed to 'misspeaking' and/or had no clinical significance may not have been verbally challenged. Moreover, as is discussed by the authors, there was an unequivocal hierarchy effect-individuals with the least 'power' (ie, low in hierarchy within the current healthcare culture) were the least likely to report the error.

This study highlights two important safety relevant questions on which I will elaborate. First, why and how should we change healthcare culture to facilitate 'speaking up'? Second, how can we best design and implement checklists and other safety interventions to yield more consistent and sustained clinician behaviour change?

\section{THE CONTINUED PROBLEM OF HIERARCHICAL CULTURE IN HEALTHCARE}

The significant influence of hierarchy on the incidence of error reporting in Muensterer et al's ${ }^{14}$ study is consistent with substantial prior evidence that lower hierarchy clinical providers are less likely to 'speak up', even when they are aware of major safety violations. ${ }^{15-17}$

Failure of a subordinate copilot to challenge or speak up to the captain in the 1977 Tenerife disaster was the impetus for the aviation industry's adoption of crew resource management (CRM). Healthcare team-training initiatives like the Agency for Healthcare Research and Quality's TeamSTEPPS now include tools such as the 'two-challenge rule' and emphasise speaking up. ${ }^{18}$ Flattened hierarchies and reliance on expertise rather than seniority, especially during crisis or stress, are an integral component of high-reliability organisations. In contrast, the persistent hierarchical culture of healthcare is anathema to positive safety attitudes and behaviours. This is particularly problematic in operating theatres where surgeons view themselves as 'captain of the ship' and where uncivil behaviour is tolerated. ${ }^{19}$ The insidious effects of hierarchy will impair effectiveness of checklist use and predispose to safety issues in all aspects of routine and emergency care. ${ }^{20}$ While team-oriented training designed to enhance the ability of lower hierarchy clinicians to 'speak up' can be effective, ${ }^{21} 22$ evidence to guide the design and implementation of these interventions is still sparse. Single training exposures have generally had limited effects, ${ }^{1723}$ in part likely due to inadequate 'potency' to achieve the desired effect ${ }^{24}$ in a clinical environment contaminated by the hierarchical culture and in part because most interventions have focused on 'assertiveness' training for the less powerful members of the team rather than, or in addition to, sensitivity or receptivity training of the most powerful (eg, surgical attendings). ${ }^{17}$

Discussions of power hierarchy to date have largely focused on clinicians' professional roles (ie, nurse vs physician) and level of experience (eg, resident vs attending). Even with two attending physicians, for example, a surgeon and anaesthesiologist, power dynamics can degrade communication and decrease team performance. In a multicentre study of experienced anaesthesiologists managing simulated crisis events, the anaesthesiologists' failure to challenge the surgeon to initiate life-saving interventions (eg, to open the abdomen in the presence of an enlarging retroperitoneal haematoma during laparoscopic surgery, or to halt surgery to cardiovert an unstable patient) was associated with lower overall scenario performance scores as determined by trained blinded anaesthesiologist video raters. ${ }^{25}$

In fact, hierarchy is much more complex and this may explain in part the variable and generally weak results seen in 'speaking up' intervention studies to date. When considering hierarchical effects on communication assertiveness, one must also consider individual characteristics including gender, race/ ethnicity, language, personal cultural background and personality, as well as the personality of those in higher power roles, microclimate factors of the team and care unit, and overall organisational culture. ${ }^{17} 22$ An interesting direction for future study is the facilitation of more positive communication (eg, expressions of gratitude or encouragement). ${ }^{26}$

In a single-site intervention study to improve the quality of handovers from anaesthesia professionals to postanaesthesia care unit (PACU) nurses, ${ }^{27}$ simulationbased training emphasised specific dyadic communication behaviours-assertiveness for the nurses when their needs were not being met and 'sensitivity' (or receptiveness) for the anaesthesia professionals when the nurses raised concerns. In poststudy interviews, this behavioural focus was considered an important contributor to the resulting sustained improvement in the quality of actual handovers. As part of this study, we explicitly taught participants to CUSS. CUSS is a graduated approach to facilitate speaking up; the acronym stands for 'I'm Concerned', 'I'm Uncomfort-

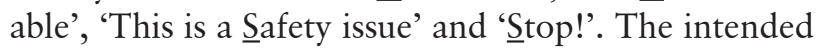
learners were taught these 'triggers' for eliciting desired behaviours (ie, to stop what they are doing and have a conversation with the initiator) and this approach creates an environment where the initiating individual can receive support from others who overhear the conversation-'Doctor, I hear that Maria is CUSSing at you? How can I help to resolve this situation?' Such 
a graded assertiveness approach to 'stop the line', developed in other industries, is increasingly being used throughout healthcare. ${ }^{28}$

\section{DESIGNING AND IMPLEMENTING MORE EFFECTIVE SAFETY TOOLS AND PROCESSES}

SSCs are just one tool used to advance overall perioperative system safety. Similarly, in commercial aviation, checklists are one tool used as part of CRM to assure operational safety. CRM is a philosophy or construct that includes explicit values and principles, procedures supported by purpose-designed checklists and other tools, and regularly scheduled mandatory simulation-based training and assessment that together contribute to an existing safety culture in pilots and across the organisation. ${ }^{29} \mathrm{CRM}$ and most of the existing aviation safety system were iteratively designed by pilots (the front-line workers) in collaboration with other stakeholders (including regulators). Healthcare must employ similar human-centred design approaches to re-engineer our safety systems.

For commercial aviation to be completely safe, no planes would fly. Similarly, safety will never be the foremost system objective in healthcare; the primary goal is to efficiently deliver cost-effective care. Instead, in any highconsequence industry, safety is a desirable by-product (an 'emergent feature') of a system designed to achieve primary operational goals. In healthcare, sick patients must be treated and there is inherent risk in doing so. ${ }^{30}$ Achieving societally acceptable levels of safety will stem from a deliberately designed system founded on a strong safety culture and truly committed leadership.

With this as background, it is not surprising that so many hospitals struggle to garner reliable and sustained benefit from the use of checklists and other safety tools. To understand what is required, I would like to draw parallels with anaesthesiology's experience of implementing another type of checklist.
The Food and Drug Administration Anesthesia

\section{Machine Pre-Use Checklist}

The earliest checklist used in healthcare to reduce adverse events is the anaesthesia equipment preuse checklist, developed in 1987 by the US Food and Drug Administration (FDA) in collaboration with the Anesthesia Patient Safety Foundation and the American Society of Anesthesiologists. ${ }^{31}$ After more than three decades of use, lessons learnt from the use of the FDA checklist parallel more recent experiences with SSCs, and are instructive to a more general understanding of the role of safety tools in healthcare (see table 1).

\section{A checklist alone is insufficient to achieve optimal results}

Hospitals that get the best results from an SSC implementation are often well-resourced organisations that already have safety-oriented committed leadership, a strong safety culture, educated and engaged front-line clinicians and an established track record of successfully implementing other safety interventions. ${ }^{32}$ That said, any hospital, given adequate commitment, resources and expertise, can implement an SSC or other substantive safety intervention successfully. In doing so, it will educate and engage its workers, improve its safety culture and set the stage for further safety and quality improvements.

A multimodal approach to safety interventions is more effective. Hospitals that were able to successfully implement all three components of the SSC saw greater reductions in postoperative complications. ${ }^{33}$ Similarly, the combination of the SSC with a complementary approach that more fully addresses preoperative and postoperative issues, the Surgical Patient Safety System, was associated with better postoperative outcomes than use of the WHO SSC alone. ${ }^{34}$ The most effective interventions are those that are based on an integrated conceptual framework and

\section{Table 1 Lessons learnt from 30 years of personal experience with and reflection about the Anesthesia Equipment Pre-Use Checklist*

$\begin{array}{ll}1 & \text { Poorly designed checklists will be less likely to be used and more error prone. } \\ 2 & \text { It is critical to involve those who are going to be using the checklist in its design and iterative refinement. } \\ 3 & \text { Checklists need to be promulgated as useful cognitive or decision aids, not as tools to enhance compliance with mandated procedures. } \\ 4 & \text { A checklist that is poorly implemented or not well integrated into workflow will have low or variable levels of adherence. } \\ 5 & \begin{array}{l}36 \\ \text { Successful checklist implementation will depend on whether its use is adequately aligned with the users' values, priorities and mental model of } \\ \text { their everyday work. }\end{array} \\ 6 & \text { Self-reported or documented adherence (consistently high with the FDA checklist) is always higher than actual observed adherence. } \\ 7 & \text { Use of (well-designed) computerisation of the checklist generally improves but does not assure adherence. }{ }^{37} \\ 8 & \begin{array}{l}\text { Even with 100\% adherence, checklist benefit will diminish if users simply go through the motions or are not fully engaged. This can be due to many } \\ \text { factors including inadequate buy-in, poorly understood leadership mandates, power dynamics, distractions and competing priorities. }\end{array} \\ 9 \text { Due to myriad contextual or confounding factors as well as challenges with study design, there will often be variable evidence of effectiveness, even } \\ \text { across well-designed randomised controlled studies (eg, 31). } \\ \begin{array}{l}\text { Despite all of the above, with incremental iterative improvements in the checklist and associated processes and technology over time, the } \\ \text { problem(s) the checklist was intended to address will steadily improve as long as that remains a system priority. For anaesthesiology, FDA checklist } \\ \text { use has been accompanied by: (A) improvements in the underlying machine technology; (B) automated self-checking of many checklist items; (C) } \\ \text { inclusion of technicians to assist in assuring that items are proactively addressed; and (D) a culture that strives to prioritise safety over production. }\end{array}\end{array}$

*This list applies equally well to other safety tools and processes including computer-based decision support systems.

FDA, Food and Drug Administration. 
follow human factor principles, especially when the safety goals are multiple or diverse. ${ }^{35}$

In our PACU handover improvement project mentioned earlier, ${ }^{27}$ the multimodal intervention produced a fourfold improvement in observed clinician behaviours (ie, conduct of actual handovers) that was sustained for at least 3 years after the intervention ceased. The project began by getting perioperative leadership buy-in, conducting observations of the current handover process and engaging front-line clinicians in all phases of study development. The criteria for an 'acceptable handover' were chosen by an independent team of clinicians. Front-line clinicians first completed a multimedia introductory webinar that included key principles and a knowledge assessment. To attend the 2-hour simulation training session, both anaesthesia professionals and PACU nurses were relieved from regular clinical duties (a strong message that this was an organisational priority). A custom patient-specific electronic form was available at every bedside in the PACU to reinforce the training during actual handovers. Performance feedback was provided to individuals, units and perioperative leadership. The number of components needed for successful safety interventions will depend on the behaviour change desired, the existing safety culture, current experience and expertise of the intended end users and the priority articulated by organisational leaders. Regardless, design and implementation must be based on a solid conceptual framework, consider the full life-cycle of the intervention (from conceptualisation to obsolescence) and employ human factors engineering and implementation science principles and tools. ${ }^{13}$

\section{CONCLUSION}

Checklists and other safety tools are potentially valuable tools to advance perioperative safety. However, when used in isolation or implemented incorrectly, checklists have significant limitations. Safety initiatives that take a systems-oriented multimodal approach to design and implementation can, with organisational leadership and determination, produce both targeted and more general safety improvement.

\section{Twitter Matthew B Weinger@VUMC_anes}

Funding The authors have not declared a specific grant for this research from any funding agency in the public, commercial or not-for-profit sectors.

Competing interests None declared.

Patient consent for publication Not required.

Provenance and peer review Commissioned; internally peer reviewed.

\section{ORCID iD}

Matthew B Weinger http://orcid.org/0000-0001-8897-2688

\section{REFERENCES}

1 Thomas EJ, Studdert DM, Burstin HR, et al. Incidence and types of adverse events and negligent care in Utah and Colorado. Med Care 2000;38:261-71.

2 Leape LL, Brennan TA, Laird N, et al. The nature of adverse events in hospitalized patients. Results of the Harvard medical practice study II. N Engl J Med 1991;324:377-84.
3 Haugen AS, Sevdalis N, Søfteland E. Impact of the world Health organization surgical safety checklist on patient safety. Anesthesiology 2019;131:420-5.

4 Haugen AS, Søfteland E, Almeland SK, et al. Effect of the world Health organization checklist on patient outcomes: a stepped wedge cluster randomized controlled trial. Ann Surg 2015;261:821-8.

5 Haynes AB, Weiser TG, Berry WR, et al. A surgical safety checklist to reduce morbidity and mortality in a global population. N Engl J Med 2009;360:491-9.

6 Pickering SP, Robertson ER, Griffin D, et al. Compliance and use of the world Health organization checklist in U.K. operating theatres. Br J Surg 2013;100:1664-70.

7 Russ S, Rout S, Caris J, et al. Measuring variation in use of the who surgical safety checklist in the operating room: a multicenter prospective cross-sectional study. J Am Coll Surg 2015;220:1-11.

8 Gawande A. The Checklist Manifesto: How to Get Things Right. New York: Metropolitan Books, 2009.

9 Catchpole K, Russ S. The problem with checklists. BMJ Qual Saf 2015;24:545-9.

10 Urbach DR, Govindarajan A, Saskin R, et al. Introduction of surgical safety checklists in Ontario, Canada. N Engl J Med 2014;370:1029-38.

11 Rydenfält C, Ek Åsa, Larsson PA. Safety checklist compliance and a false sense of safety: new directions for research. BMJ Qual Saf 2014;23:183-6.

12 Anthes E. The trouble with checklists. Nature 2015;523:516-8.

13 Burian BK, Clebone A, Dismukes K, et al. More than a tick box: medical checklist development, design, and use. Anesth Analg 2018;126:223-32.

14 Muensterer OJ, Kreutz H, Poplawski A, et al. Timeout procedure in paediatric surgery: effective tool or lip service? a randomised prospective observational study. BMJ Qual Saf 2021;30:622-7.

15 Bould MD, Sutherland S, Sydor DT, et al. Residents' reluctance to challenge negative hierarchy in the operating room: a qualitative study. Can J Anaesth 2015;62:576-86.

16 Greenberg CC, Regenbogen SE, Studdert DM, et al. Patterns of communication breakdowns resulting in injury to surgical patients. J Am Coll Surg 2007;204:533-40.

17 Weller JM, Long JA. Creating a climate for speaking up. $\mathrm{Br} J$ Anaesth 2019;122:710-3.

18 Agency for Healthcare Research and Quality (AHRQ). The team strategies and tools to enhance performance and patient safety (TeamSTEPPS) program. Rockville, MD: US Department of Health and Human Services, 2021.

19 Katz D, Blasius K, Isaak R, et al. Exposure to incivility hinders clinical performance in a simulated operative crisis. BMJ Qual Saf 2019;28:750-7.

20 Sydor DT, Bould MD, Naik VN, et al. Challenging authority during a life-threatening crisis: the effect of operating theatre hierarchy. Br J Anaesth 2013;110:463-71.

21 Omura M, Maguire J, Levett-Jones T, et al. The effectiveness of assertiveness communication training programs for healthcare professionals and students: a systematic review. Int J Nurs Stud 2017;76:120-8.

22 Kim S, Appelbaum NP, Baker N, et al. Patient safety over power hierarchy: a scoping review of healthcare professionals' Speaking-up skills training. J Healthc Qual 2020;42:249-63. 
23 Oner C, Fisher N, Atallah F, et al. Simulation-based education to train learners to "speak up" in the clinical environment: Results of a randomized trial. Simul Healthc 2018;13:404-12.

24 Weinger MB. The pharmacology of simulation: a conceptual framework to inform progress in simulation research. Simul Healthc 2010;5:8-15.

25 Weinger MB, Banerjee A, Burden AR, et al. Simulation-Based assessment of the management of critical events by boardcertified anesthesiologists. Anesthesiology 2017;127:475-89.

26 Riskin A, Bamberger P, Erez A, et al. Expressions of gratitude and medical team performance. Pediatrics 2019;143:e20182043.

27 Weinger MB, Slagle JM, Kuntz AH, et al. A multimodal intervention improves Postanesthesia care unit handovers. Anesth Analg 2015;121:957-71.

28 Green B, Oeppen RS, Smith DW, et al. Challenging hierarchy in healthcare teams - ways to flatten gradients to improve teamwork and patient care. Brit J Oral Maxillofac Surg 2017;55:449-53.

29 Helmreich R, Merrit A. Safety and error management: The role of crew resource management. In: Hayward B, Lowe A, eds. Aviation resource management. Aldershot, UK: Ashgate, 2000: 107-19.

30 Bagian JP. How safe is safe enough for space and health care?: communication and acceptance of risk in the real world. JAMA Neurol 2019;76:399-401.
31 March MG, Crowley JJ. An evaluation of anesthesiologists' present checkout methods and the validity of the FDA checklist. Anesthesiology 1991;75:724-9.

32 Abbott T, Ahmad T, Phull M. International surgical outcomes study (ISOS) group. the surgical safety checklist and patient outcomes after surgery: a prospective observational cohort study, systematic review and meta-analysis. Br J Anaesth 2018;120:146-55.

33 Mayer EK, Sevdalis N, Rout S, et al. Surgical checklist implementation project: the impact of variable who checklist compliance on risk-adjusted clinical outcomes after national implementation: a longitudinal study. Ann Surg 2016;263:58-63.

34 Storesund A, Haugen AS, Flaatten H, et al. Clinical efficacy of combined surgical patient safety system and the world Health organization's checklists in surgery: a nonrandomized clinical trial. JAMA Surg 2020;155:562-70.

35 McCulloch P, Morgan L, New S, et al. Combining systems and teamwork approaches to enhance the effectiveness of safety improvement interventions in surgery: the safer delivery of surgical services (S3) program. Ann Surg 2017;265:90-6.

36 Smith NT, Smith B, et al. Performance of the pre-anesthetic equipment checkout: a simulator study. J Clin Monit Comput $1999 ; 15: 233-4$.

37 Jelacic S, Bowdle A, Nair BG, et al. The effects of an aviationstyle computerised pre-induction anaesthesia checklist on pre-anaesthetic set-up and non-routine events. Anaesthesia 2019;74:1138-46. 